\title{
The Study of 3D Reaching Tasks on Visual Servoing Using Quaternion
}

DOI : 10.36909/jer.12031

\author{
Liucun Zhu ${ }^{*, * *}$, Junqi Luo ${ }^{*, * *}$, Mingyou Chen ${ }^{* *}$, Haofeng Deng ${ }^{* *}$ \\ * College of Mechanical Engineering, Guangxi University, Nanning 530004, Guangxi, China \\ ** Advanced Science and Technology Research Institute, Beibu Gulf University, Qinzhou, \\ 535000, Guangxi, China.
}

**Email: 2011401005@st.gxu.edu.cn; Corresponding Author: Junqi Luo

\begin{abstract}
The image-based monocular visual servoing is difficult to realize the grasping task on threedimension. This study presented a method of quaternion on 3D reaching tasks of visual servoing. This method used a camera and a laser scanner to acquire the information of the images and the depth. Then the parameters of Jacobian matrix could be determined by the method of the quaternion. A particular constraint mechanism was also proposed to optimize the reaching trajectory of the robot. The simulation results demonstrated our proposed method could implement the $3 \mathrm{D}$ reaching tasks successfully and had a significant performance improvement compared to conventional methods.
\end{abstract}

Key words: image-based visual servoing; monocular vision; reaching task; quaternion. 


\section{INTRODUCTION}

The image-based visual servoing (IBVS) system based on monocular vision is used only one camera and has the advantages of low-cost and simple configuration (Tsai et al., 2017; Mcfadyen et al., 2016; Ishiyama et al., 1999). However, the monocular vision is difficult to get the depth information in the actual application. But then again, the stereo visions, e.g., binocular and multocular visions, are also difficult to capture the image feature of the object by using all cameras at the same time. Also, the cost of using the stereo vision is higher than the monocular vision system, or the system combined the CCD camera and the laser scanner. Therefore, the scheme of the camera-laser system seems like a better selection, considering both the cost and the performance (Grosso et al., 1996; Malis et al., 1999). In general, the image Jacobian uses a constant value for many systems of the image-based visual servoing, which might cause the system to fail to converge and reach the target position. Investigations from some literature verified that the optimal trajectory in the Cartesian Space could be realized more easily by position-based visual servoing (PBVS), but such a method is highly dependent on the calibration accuracy and has huge executing time (Fathian et al., 2018). To avoid this problem, Mail et al. have proposed a new technique called 2-1 / 2-D visual servoing (HVS). The method uses a Homography matrix according to the relation between the depth of the image Jacobian and the distance from the frame of the camera origin to the reference plane of the object to on-line estimate the 3D depth. However, such approaches, its computational complexity on the determination of homography matrix is large, which leads to the poor realtime performance (Fujimoto et al., 2000; Sun et al., 2018; Liu et al., 2019).

In general, a calibration operation needs to be done before the visual servo task, which is time-consuming manual work (Horaud et al., 1995). Theoretically, PBVS and HVS are not suitable for developing uncalibrated visual servos. $\mathrm{Hu}$ et al., developed quaternion parameterization to realize state estimating and measuring closed-loop system, and through theory, Lyapunov-based analysis and simulation experiments, verified that IBVS can realize 
servo control under the condition that camera calibration parameters were unknown. Nonetheless, IBVS is notoriously hard to predict the 3D motion of the camera or the robot because the 3D trajectory generation is considered by only using the deviations between the initial and the goal features into the minimum in the image plane. Besides, the object might disappear from the view of the camera in the worst case of reaching task, which is a disorder characterized by trajectory control. For this issue, some scholars put forward solutions. Ishiyama et al. proposed an approach of image-based visual servoing for the optimal 3D trajectory by using Homography and epipolar geometry. For the general case, the feature point of an object is on a reference plane, a projection Homography matrix is used to optimize the trajectory. Grosso et al. proposed a controlling method to generate the optimal trajectory for $3 \mathrm{D}$ reaching tasks by using binocular vision and measuring the movement of a robot hand continuously.

Shen et al. employed a quaternionic quadratic polynomials to optimize the motion path of the camera on the tracking problems of moment-based features of cylinders.

Keshmiri and Xie proposed a semi-offline trajectory planning algorithm for the eye-in-hand application of IBVS, showed an excellent robust in the task which requires a big angle rotation of the camera on its center.

The function of stable tracking control (Kara T and Mary A H, 2018) accomplished by visual servoing is a challenging but critical issue until now. Thus, the robotic tracking control problem, especially in the 3D reaching task, has been extensively focused on by researchers (Gordon et al., 1988; Lee et al. 2016; Wang et al., 2019; Guo et al., 2020; Dirik M et al., 2020). Kosmopoulos (2011) carried out an uncalibrated Jacobian matrix estimation method of visual servo for applying to the semi-structures production line. This method is similar to ours, that is, the identification of the Jacobian matrix can be realized without the depth information of the image.

Chen et al. (2018) aimed at the drawbacks of the traditional Jacobian-matrix-pseudo-inverse 
(JMPI) method, which requires the robot parameter model and the position error accumulation to be known. The jacobian matrix-adaption (JMA) method with the Zeroing dynamics was proposed. The effectiveness of the proposed method was verified by comparative experiments and robustness tests. Yang et al. (2019) proposed an improved Lyapunov-based control law of IBVS for the UAV's fast-moving tracking. this uses centroid coordinates as an input to avoid pseudo-inverse of the Jacobian matrix. Therefore, it is easier to obtain time-saving control output. Zhou et al. (2019) used the Kalman filter (KF) to estimate the Jacob matrix, which improves the anti-noise ability of the system. Further, an LSTM model was used to predict the observed value of the Jacob matrix of the image, thereby solving the problems of difficulty KF model initializing and low accuracy of Jacob matrix estimation. The simulation results show that the method has better noise tolerance and convergence on speed. Also, aiming at the problem of automatic assembly, Gu et al. (2020) established a combination of Kalman filter and smooth variable structure filter to improve projective homography and realize online estimation of the Jacobian matrix. The results showed good anti-interference and real-time performance. Li and Xiong (2021) reported an HVS control method for the path planning on the tracking task of the mobile manipulator robot, considering both the mobile robot and the arm simultaneously. The Kalman filter is then used to redress the pose of the end-effector. The better tracking effect is verified by a comparison experiment.

In our research, a visual servoing system for catching a moving object is established, aiming to implement stable grasping tasks. The system is configured by a special sensor, which consists of a laser stripe generator fixed rigidly to the CCD camera and projects planar light on the polyhedral object. The CCD camera with laser sensor mount on a robot end-effector to accomplish the $3 \mathrm{D}$ reaching tasks. When we consider the grasping action, the action can be roughly classified into visual reach and grasp movements. In this paper, a 3D reaching task is focused. It is necessary to adopt the line features of instead of point features in the visual servoing control system, aiming to expand the reaching task to the grasping task. For 
example, the straight line, which connects the contacting point of a robot's 2 fingers, is related to grasp force. In order to improve the tracking performance, the quaternion-based visual servo method is implemented. Firstly, the quaternion is used to estimate the displacement between a CCD camera and an object, and to estimate the 3D data of image Jacobian. Secondly, the approach is to constrain the robot moves along the optimal trajectory, which would be beneficial to avoid the respective drawbacks of classical position-based and imagebased visual servoing. Unlink the position-based visual servoing, it does not require the 3D geometric model of the object, and also unlink the image-based visual servoing, the 3D data of image Jacobian can be estimated. The main contributions are summarized as follows:

(1) Theoretically analyze and implement the online identification of the Jacobian matrix using quaternion and depth information.

(2) Propose the quaternion method to optimize the camera's 3D trajectories.

(3) We propose a constraint method to eliminate the deviation on the camera's field of view caused by low camera resolution.

This paper is organized as follows: Section 2 introduced the visual reaching model. Section 3 introduced the motion representing and rotation matrix of line. Section 4 analyzed the estimation of the image Jacobian of line. Section 5 proposed feedback control and parameter estimation. At the end of the article, we will make a summary.

\section{VISUAL REACHING MODEL}

\section{System and Line Representation}

As shown in Fig 1, the laser light striped generator configures an optical plane by using a light ray, which is perpendicular to the optical axis of a pipe-like lens and spreads in the shape of a fan and called the light plane $\pi_{\mathrm{s}}$. It is expressed with the following equation.

$$
a_{s} x+b_{s} y+c_{s} z+d_{s}=0 \#(1)
$$


Correspondently, the polyhedron surface where the laser stripes were projected and called the striped plane $\pi_{\mathrm{i}}$, expressed the following expression.

$$
a_{i} x+b_{i} y+c_{i} z+d_{i}=0(i=0,1,2, \ldots) \#(2)
$$

where $a_{i}, b_{i}, c_{i}$, and $d_{i}$ are the 3D parameters of the striped plane on the surface of polyhedron. In this study, the research is focused on the polyhedron whose surface is a plane.

The intersection curve between an optical plane and the partial surface in polyhedron turns into a straight line and calls the 3D laser stripe line. The straight line projected the 3D laser line on the image plane is expressed by straight-line parameters $\theta$ and $\rho$, as shown in the following equation.

$$
\rho=u \sin \theta+v \sin \theta \#(3)
$$

Where the coordinates $(\mathrm{u}, \mathrm{v})$ are belonging to the straight line, $\rho$ is the perpendicular distance from the center to the straight line in the image plane, and $\theta$ is an angle of the altitude and vaxis (horizontal axis).

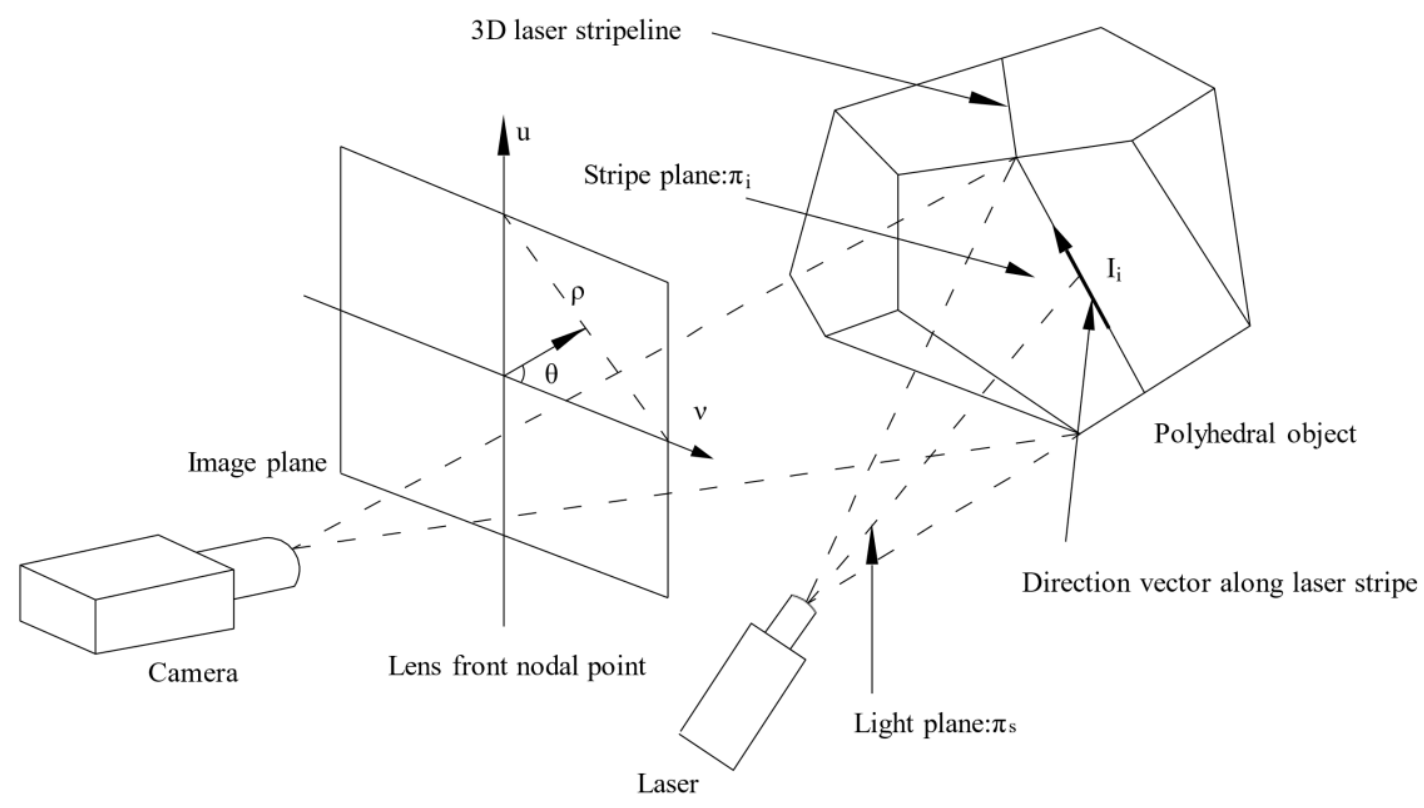

Figure 1 system structure of the CCD camera and laser scanner. 
Assume the projective geometry of the camera is modeled by perspective projection, and the coordinates of point $\mathrm{P}=(\mathrm{x}, \mathrm{y}, \mathrm{z})^{\mathrm{T}}$ are expressed with the camera coordinate frame $\mathrm{R}_{\mathrm{c}}$. hence, the projective geometry will project onto the image plane with coordinates $p=(u, v)^{\mathrm{T}}$, the expression is shown below.

$$
\left[\begin{array}{l}
u \\
v
\end{array}\right]=\frac{f}{z}\left[\begin{array}{l}
x \\
y
\end{array}\right] \#(4)
$$

Where $\mathrm{f}$ is the focal length of the camera lens.

\section{Inverse Projection and Direction of Line}

The coordinates of the image plane and the light plane, the point coordinates of the stripe plane in 3D space can be calculated (Keshmiri et al., 2016) by using the function of the calibration parameters of the camera. According to the relation of coordinate $\mathrm{z}$ and coordinates $\mathrm{x}$ and $\mathrm{y}$, we can obtain the following expression.

$$
z=z_{0}+\tan \alpha(y+x \tan \beta) \#(5)
$$

where $\mathrm{z}_{0}$ is the intersection of the camera's optical axis and the optical plane on the calibration plane and meet $\mathrm{z}_{0}=\mathrm{y}_{0 *} \tan \alpha$. The values of three parameters the disparity angle $\alpha$, a tilt angle $\beta$, and an offset $\mathrm{Y} 0$, should be determined during the calibration. The parameters are produced by the laser-stripe sensor rigidly attached to the camera reference frame, concerning a coordinate frame defined by the camera image plane. The following expression can be derived from the combination of equations (4) and (5) using the inverse projective technique.

$$
\left\{\begin{array}{l}
x=\frac{u z_{0}}{1-(v+u \tan \beta) \tan \alpha} \\
y=\frac{v z_{0}}{1-(v+u \tan \beta) \tan \alpha} \#(6) \\
z=\frac{z_{0}}{1-(v+u \tan \beta) \tan \alpha}
\end{array}\right.
$$


The direction vector of the 3D laser line which is on a polyhedron in 3D space can be obtained from equations (3) and (6).

$$
\begin{gathered}
l_{i}=\left(l, q_{i}, q_{i} \tan \alpha\right)^{T} \#(7) \\
q_{i}=\frac{-\cos \theta_{i}-P_{i} \tan \alpha \tan \beta}{\sin \theta_{i}+P_{i} \tan \alpha} \#(8)
\end{gathered}
$$

\section{MOTION REPRESENTING AND ROTATION MATRIX OF LINE \\ Homography Matrix of Image Point}

Assume one of the planes of grasping (e.g. a contact surface of a finger and an object) is one surface of a polyhedron which is the stripe plane in a 3D laser line. Hence, the 3-D reaching task is to control the motion of the robot hand from the present position to the plane of grasping as the target position. In this section, the Homography matrix is analyzed to obtain the rotational matrix and the translational vector.

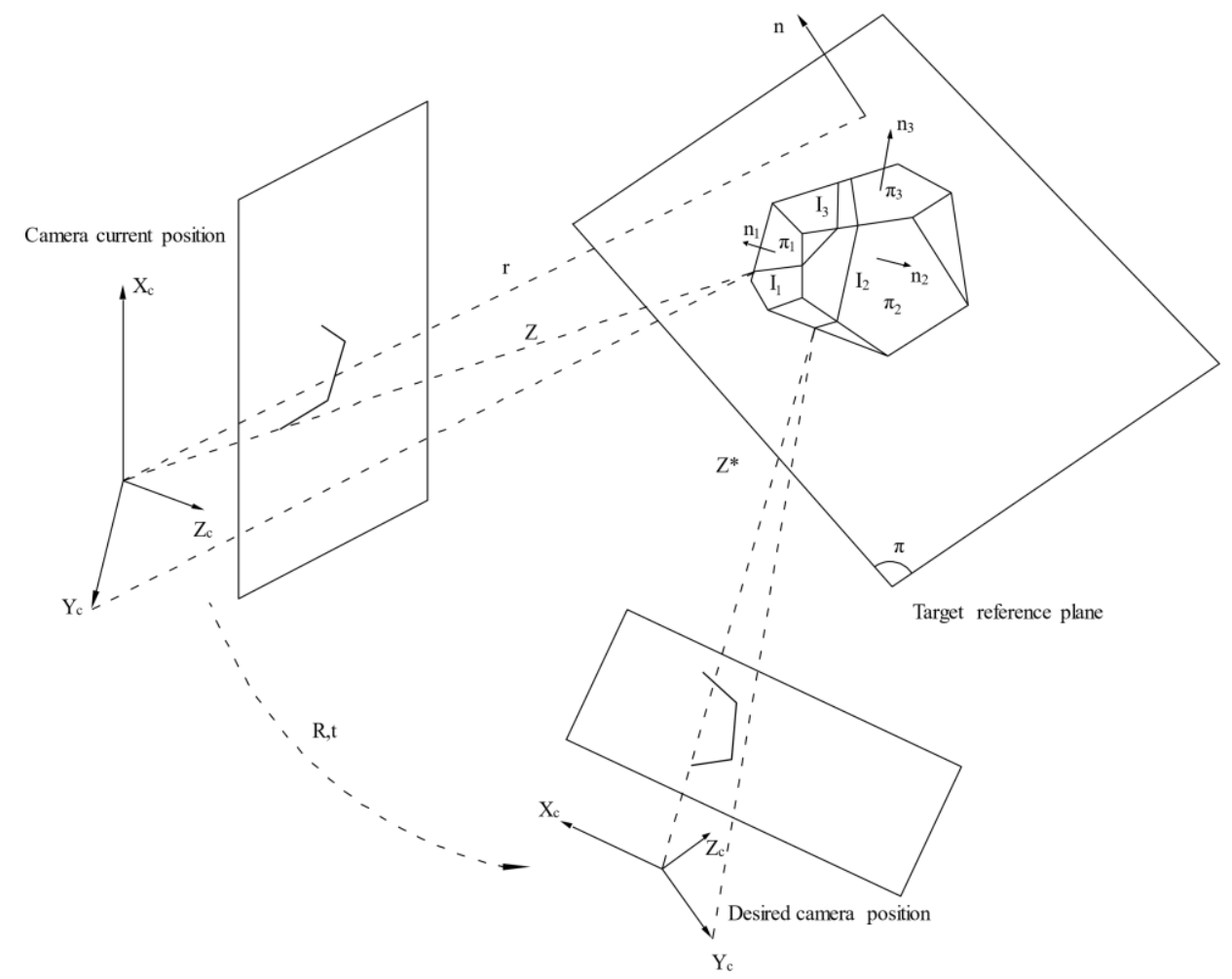

Fig 2 Three line segments of laser stripe on camera and image frames, generated by the 
intersection of a light plane and the surface of a polyhedral object.

Fig 2 shows the relation between the camera's position and the target position at the current time. The relation can be expressed by using the rotational matrix and the translational vector. Besides, the reference plane that the object exists can be determined by using a normal vector of a plane and the distance from the plane to the image plane in the camera frame. Let the normal vector be $\mathrm{n}^{*}$, the rotational matrix be $\mathrm{R}$, and let the translational vector be $\mathrm{t}$, then the relation between the current position $\mathrm{P}$ and the target position $\mathrm{P}^{*}$ can be expressed as follows:

$$
\begin{gathered}
P=R P^{*}+t \#(9) \\
P=\left(R+\frac{t}{r^{*}} n^{* T}\right) P^{*} \#(10)
\end{gathered}
$$

where $r^{*}=n^{* T} P^{*}$ is the distance from the target position to the reference plane. The current position $\mathrm{P}$ can be also expressed by the following expression after combining equations (4) (10).

$$
\begin{gathered}
p=\frac{z^{*}}{z}\left(R+\frac{t}{r^{*}} n^{* T}\right) p^{*} \#(11) \\
p=C P^{*} \#(12)
\end{gathered}
$$

where $\mathrm{C}$ is a $3 \times 3$ matrix, called the Collineation matrix or the projective Homography matrix. In the case that the object is on a plane, the $\mathrm{C}$ matrix can be determined by at least 4 known points, on the basis of the linear transformation between the present and target position. However, in the case that the object is a $3 \mathrm{D}$ polyhedron, the determination of Homography matrix becomes a nonlinear problem. In order to identify the Homography matrix, more than 8 coordinate points are needed (Three points lie on the plane, and the other five points don't belong to the plane). The 3D homography will be described in the next section.

\section{D Homography}


Let the point $\mathrm{m}$ be the point on the image plane with the camera frame, and the matrix of the interior parameters in camera $\mathrm{W}$ be the transformation matrix between the standardized coordinates and the pixel coordinates in the image frame. The relation between the point $\mathrm{m}$ in the camera frame and the point $\mathrm{p}$ in the image frame can be written as follows.

$$
\boldsymbol{p}=\boldsymbol{W} \boldsymbol{m}=\left[\begin{array}{ccc}
\alpha_{u} & 0 & u_{0} \\
0 & \alpha_{v} & v_{0} \\
0 & 0 & 1
\end{array}\right] \#(13)
$$

where $\alpha_{\mathrm{u}}, \alpha_{\mathrm{v}}, \mathrm{u}_{0}$ and $\mathrm{v}_{0}$ are the camera interior parameters. $\alpha_{\mathrm{u}}$ and $\alpha_{\mathrm{v}}$ are the product of the unit scaling factor and the focal distance in the perpendicular and the horizontal directions [pixels $/ \mathrm{mm}$ ] of the image frame. The parameters $\mathrm{u} 0$ and $\mathrm{v} 0$ represent the center of the image plane. If the camera parameters are known, the 3D Homography matrix $\mathrm{H}$ can be calculated as follows.

$$
\boldsymbol{H}=\mathbf{R}+\left(\frac{\boldsymbol{t}}{r^{*}}\right) \boldsymbol{n}^{* T} \#(14)
$$

Assume the $\mathrm{H}$ is given, the rotational matrix $\mathrm{R}$ and translational vector $\mathrm{t}$ can be computed by using the decomposed homography matrix $\mathrm{H}$.

\section{From Line Representation to Point Representation}

The rotational matrix has been obtained by using the method of point coordinates according to the previous section. However, the laser projection system can only use the line segment instead of the points. is used in such. Hence, it is necessary to develop a method that calculated the rotational matrix by using the line. According to Fig 3, let the direction vector of the line be $\mathrm{l}_{3 \mathrm{D}}$, the normal vector of the current light plane be $\mathrm{n}_{3 \mathrm{D}}$ and the normal vector of the target light plane be $\mathrm{n}_{3 \mathrm{D}}{ }^{*}$, and then the following relation can be derived.

$$
\boldsymbol{n}_{3 D}=\boldsymbol{H} \boldsymbol{n}_{3 D}^{*} \#(15)
$$




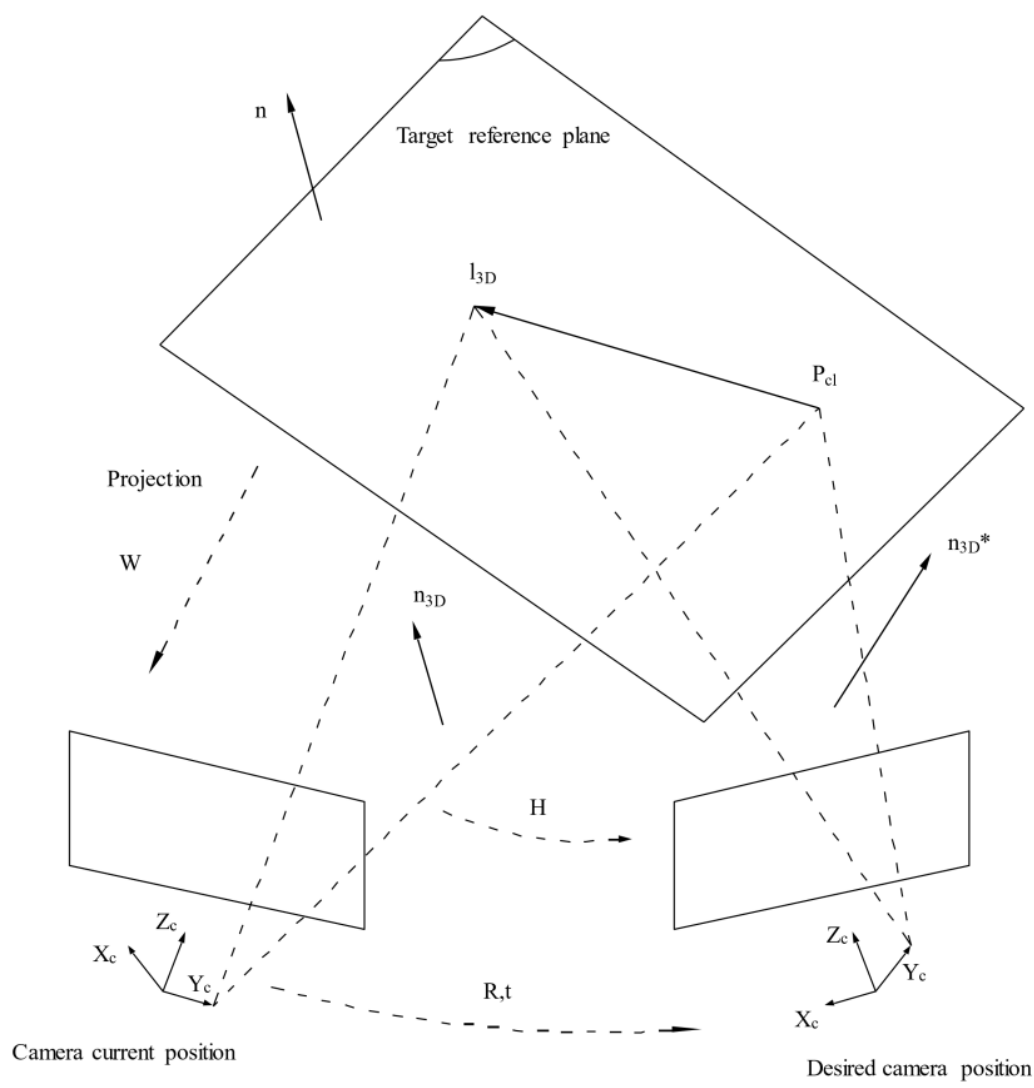

Fig 3 The elements used in the definition of lines in motion

If the positions of the camera and laser generator are determined, the coordinates $\mathrm{P}_{\mathrm{cl}}$ can be calculated. By using the reverse projection, the direction vector of the line $1_{3 \mathrm{D}}$ can be obtained. Therefore, the normal vector of the current light plane $\mathrm{n}_{3 \mathrm{D}}$ and the normal vector of the target light plane n3D* can be obtained [17]. The equations (15) and (16) shows the homography matrix $\mathrm{H}$ can be calculated by using the line feature.

$$
\left\{\begin{array}{l}
\boldsymbol{n}_{3 D}=\boldsymbol{P}_{c l} \times \boldsymbol{l}_{3 D} \\
\boldsymbol{n}_{3 D}^{*}=\boldsymbol{P}_{c l}^{*} \times \boldsymbol{l}_{3 D}^{*}
\end{array}\right.
$$

\section{ESTIMATION THE IMAGE JACOBIAN OF LINE}

\section{Normal Estimation Using Quaternion}

Firstly, $\mathbf{n}_{\mathrm{i}}=\left(a_{i}, b_{i}, c_{i}\right)^{\mathrm{T}}$ is expressed as the normal vector of the grasping plane in the current time, the direction vector of the $3 \mathrm{D}$ laser line is expressed as $\mathbf{l}_{\mathbf{i}}$. The normal vector is perpendicular to the direction vector, shown in the expression below. 


$$
\boldsymbol{n}_{i} \cdot \boldsymbol{l}_{i}=0 \#(17)
$$

The product calculation of quaternion can be shown in equation (18), then the vector can be expressed (Tian et al., 2017) in equation (19). In addition, the rotational motion can be expressed by using the quaternion, shown in (20).

$$
\begin{gathered}
\boldsymbol{n}_{i} \cdot \boldsymbol{l}_{i}=-\frac{1}{2}\left(\boldsymbol{n}_{i} \boldsymbol{l}_{i}+\boldsymbol{l}_{i} \boldsymbol{n}_{i}\right) \#(18) \\
l=\left(0, \boldsymbol{l}_{i}\right), \quad n=\left(0, \boldsymbol{n}_{i}\right), \quad n^{*}=\left(0, \boldsymbol{n}_{i}^{*}\right) \#(19) \\
n=q n^{*} \bar{q} \#(20) \\
\boldsymbol{R}=\left(\begin{array}{ccc}
q_{0}^{2}+q_{1}^{2}-q_{2}^{2}-q_{3}^{2} & 2\left(q_{1} q_{2}-q_{0} q_{3}\right) & 2\left(q_{1} q_{3}+q_{0} q_{2}\right) \\
2\left(q_{1} q_{2}+q_{0} q_{3}\right) & q_{0}^{2}-q_{1}^{2}+q_{2}^{2}-q_{3}^{2} & 2\left(q_{2} q_{3}-q_{0} q_{1}\right) \\
2\left(q_{1} q_{3}-q_{0} q_{2}\right) & 2\left(q_{2} q_{3}+q_{0} q_{1}\right) & q_{0}^{2}-q_{1}^{2}-q_{2}^{2}+q_{3}^{2}
\end{array}\right) \#(21)
\end{gathered}
$$

where $\boldsymbol{l}, \boldsymbol{n}, \boldsymbol{n}^{*}$ are quaternion. $\boldsymbol{q}=\left(q_{0}, q_{1}, q_{2}, q_{3}\right)$ is the unit quaternion that obtains from the rotational matrix $\mathbf{R}$.

Combine equations (18) -(20), the following expression can be derived as (21). Then the error function can be obtained as (22) and (23), using the method of least squares.

$$
\begin{gathered}
q n^{*} \bar{q}+\operatorname{lq} n^{*} \bar{q}=0 \#(22) \\
f(\boldsymbol{n} *)=\left\|q n^{*} \bar{q} l+l q n^{*} \bar{q}\right\|^{2}=n^{* T} \boldsymbol{A} n^{*} \#(23)
\end{gathered}
$$

where $\mathbf{A}$ is a $4 \times 4$ matrix, shown in (24).

$$
\begin{gathered}
A=\left(\begin{array}{l}
\boldsymbol{W}(l) \boldsymbol{W}(q)^{T} \boldsymbol{Q}(q)+\boldsymbol{W}(q)^{T} \boldsymbol{Q}(l) \boldsymbol{Q}(q) \\
\boldsymbol{W}(l) \boldsymbol{W}(q)^{T} \boldsymbol{Q}(q)+\boldsymbol{W}(q)^{T} \boldsymbol{Q}(l) \boldsymbol{Q}(q)
\end{array}\right)^{T} \#(24) \\
\boldsymbol{Q}(t)=\left(\begin{array}{cccc}
t_{0} & -t_{1} & -t_{2} & -t_{3} \\
t_{1} & t_{0} & -t_{3} & t_{2} \\
t_{2} & t_{3} & t_{0} & -t_{1} \\
t_{3} & -t_{2} & t_{1} & t_{0}
\end{array}\right) \#(25)
\end{gathered}
$$




$$
\boldsymbol{W}(t)=\left(\begin{array}{cccc}
t_{0} & -t_{1} & -t_{2} & -t_{3} \\
t_{1} & t_{0} & t_{3} & -t_{2} \\
t_{2} & -t_{3} & t_{0} & t_{1} \\
t_{3} & t_{2} & -t_{1} & t_{0}
\end{array}\right) \#(26)
$$

In order to resolve the problem of minimization, we can use the Lagrangian multipliers, shown in (26).

$$
\min _{\boldsymbol{n}^{*}} \boldsymbol{f}\left(n^{*}\right)=\min _{\boldsymbol{n}^{*}}\left(n^{* \boldsymbol{T}} \boldsymbol{A} n+\boldsymbol{k}_{\boldsymbol{m i n}}\left(1-n^{* \boldsymbol{T}} n^{*}\right)\right) \#(27)
$$

where $\mathbf{k}_{\min }$ is the least eigenvalue of $\mathbf{A}$. Therefore, the quaternion $\boldsymbol{n}$ can be evaluated. From the above steps, we can obtain 3D data of image Jacobian of the line that is $a_{\mathrm{i}}, b_{\mathrm{i}}$ and $c_{\mathrm{i}}$ by decomposing the quaternion $\boldsymbol{n}$.

\section{Distance and Depth Estimation}

In this section, we calculate 3D data $d_{\mathrm{i}}$ in the image Jacobian by using the relation between the parameters of grasping plane in the current position and the distance in the goal position. in Fig $4, r_{i}$ is the distance from the camera coordinate origin $\mathbf{s}_{1}$ to the grasping plane in the current position, $r_{i}{ }^{*}$ is the distance from the camera coordinate origin $\mathbf{s}_{2}$ to the grasping plane in the goal position. The following relation is derived in (28). The Polyhedral parameter $d_{i}$ can be estimated in (29) due to $\mathbf{H}$ is known and the goal distance can be approximated.

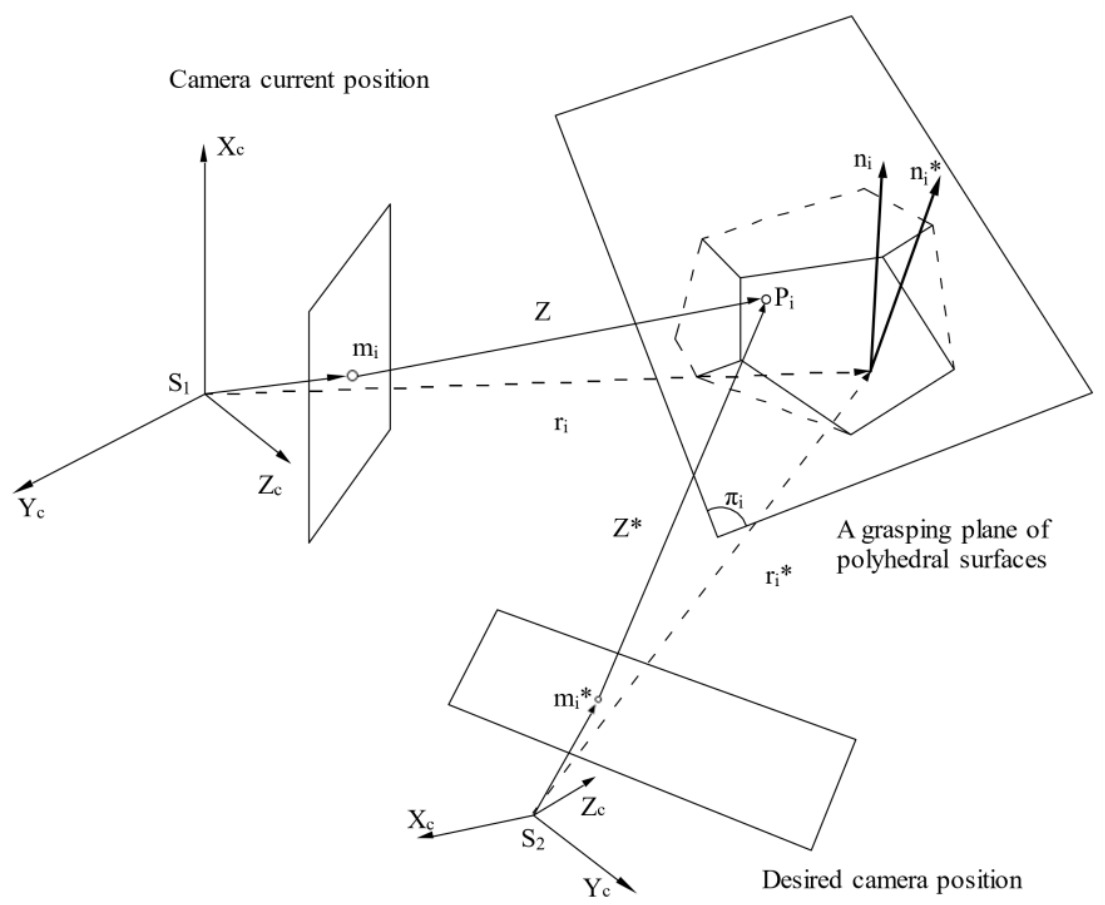


Fig 4 Polyhedral parameter $d_{i}$ is obtained by using the relation of depth and distance in the current and desired positions.

$$
\begin{gathered}
\tau=\frac{r_{i}}{r_{i}{ }^{*}}=\frac{\boldsymbol{n}_{i}^{T} \boldsymbol{s}_{1}}{r_{i}{ }^{*}}=\frac{\left(\boldsymbol{R} \boldsymbol{n}_{i}^{*}\right)^{T}\left(\boldsymbol{R} \boldsymbol{s}_{2}+\boldsymbol{t}\right)}{r_{i}{ }^{*}}=1+\frac{\boldsymbol{n}_{i}{ }^{T} \boldsymbol{t}}{r_{i}{ }^{*}}=\operatorname{det}(\boldsymbol{H}) \#(28) \\
d_{i}=-z \boldsymbol{n}_{i}^{T} \boldsymbol{m}_{i}=-r_{i}=-r_{i}{ }^{*} \operatorname{det}(\boldsymbol{H}) \#(29)
\end{gathered}
$$

where $\tau$ is the ratio of current distance $r_{i}$ and goal distance $r_{i}{ }^{*} . \mathbf{m}_{\mathrm{i}}$ is the coordinates of the image plane in the camera frame.

\section{VISUAL REACHING CONTROL}

\section{Control Scheme of Optimal Trajectory}

To prevent the object is absconded from the view of the camera, the trajectory would be restrained into the translational movement, so that camera might arrive at the target position more rapidly, the expression is shown below.

$$
\mathbf{v}=-\dot{\boldsymbol{t}} \#(30)
$$

Because the motion of the robot hand is restrained as an optimal trajectory, the derivative of the rotational matrix is $\dot{\mathbf{R}}=0$. According to the differential equation (28) and equation (30), we can obtain as

$$
\dot{\tau}=\frac{\mathbf{n}_{i}^{* T}}{r_{i}^{*}}\left(\dot{\mathbf{R}}^{T} \mathbf{t}+\mathbf{R}^{T} \mathbf{t}\right)=\frac{\mathbf{n}_{i}^{* T} \mathbf{R}^{T}}{r_{i}^{*}}\left(\mathbf{R} \dot{\mathbf{R}}^{T} \mathbf{t}+\mathbf{i}\right)=-\frac{\mathbf{n}_{i}^{T}}{r_{i}^{*}} \mathbf{v} \#(31)
$$

$\tau$ becomes 1 when the current distance $r_{\mathrm{i}}$ and the goal distance $r_{\mathrm{i}}{ }^{*}$ are equal. If the goal value of $\tau$ converge to $\tau^{*}$, which has the value of 1 , the behavior of distance ratio is found as (32)

$$
\dot{\tau}+\lambda_{\text {trans }}\left(\tau-\tau^{*}\right)=0 \#(32)
$$

The control input $\mathbf{v}$ can be computed from the following equation:

$$
\mathbf{v}=\lambda_{\text {trans }} r_{i}^{*} \frac{\mathbf{n}_{i}}{\Delta}\left(\tau-\tau^{*}\right) \#(33)
$$

where $\lambda_{\text {trans }}>0$ is the gain of translational velocity, and $\Delta=a_{\mathrm{i}}^{2}+b_{\mathrm{i}}{ }^{2}+c_{\mathrm{i}}^{2}$. consider the $(\theta, \rho)$ converges to goal value and then the expression below can be determined. 


$$
\left(\begin{array}{c}
\dot{\theta} \\
\dot{\rho}
\end{array}\right)+\lambda_{\text {rot }}\left(\left(\begin{array}{c}
\dot{\theta} \\
\dot{\rho}
\end{array}\right)-\left(\begin{array}{c}
\theta^{*} \\
\rho^{*}
\end{array}\right)\right)=0 \#(34)
$$

where $\lambda_{\text {rot }}>0$ is a gain of rotational velocity. The control input $\boldsymbol{\omega}$ can be obtained as follows

$$
\boldsymbol{\omega}=-\mathbf{J}_{\text {rot }}^{+}(\theta, \rho)\left(\lambda_{\text {rot }}\left(\mathbf{L}_{i}-\mathbf{L}_{i}^{*}\right)-\lambda_{\text {trans }} \mathbf{J}_{\text {trans }} r_{i}^{*} \frac{\mathbf{n}_{i}}{\Delta}\left(\tau-\tau^{*}\right)\right) \#(35)
$$

where $\mathbf{J}_{\text {rot }}^{+}(\theta, \rho)$ is a pseudo-inverse for $\mathbf{J}_{\text {rot }}(\theta, \rho)$.

\section{Feedback Control and Parameter Estimation}

For unknown camera parameters, the parameters can be calibrated on-line by using feedback. Firstly, the relation of presumed value $(\theta, \rho)$ from the projection relation and the preceding value ( $\left.\theta_{\text {old }}, \rho_{\text {old }}\right)$ from projection equation (4) can be expressed as follows.

$$
\begin{gathered}
\theta=\operatorname{actan}\left(\frac{\alpha_{u}}{\alpha_{v}} \tan \left(\theta_{\text {old }}\right)\right) \#(36) \\
\rho=u_{0} \cos (\theta)+v_{0} \sin (\theta)+\frac{\cos (\theta)}{\cos \left(\theta_{\text {old }}\right)} \alpha_{u} \rho_{\text {old }} \#(37)
\end{gathered}
$$

Let $\mathbf{L}$ be $(\theta, \rho)^{\mathrm{T}}$ and $\boldsymbol{\sigma}$ be $\left(\alpha_{\mathrm{u}}, \alpha_{\mathrm{v}}, u_{0}, v_{0}\right)^{\mathrm{T}}$, and then the following relation can be derived

$$
\dot{L}=\eta \mathbf{J}_{\mathrm{trans}} \mathbf{v}+\eta \mathbf{J}_{\mathrm{rot}} \boldsymbol{\omega}+\boldsymbol{\mu} \dot{\sigma} \#(38)
$$

Where

$$
\begin{aligned}
& \eta=\left(\begin{array}{cc}
\frac{\alpha_{u} \alpha_{v}}{A} & \frac{1}{A}\left\{\alpha_{u} v_{0} \cos \theta-u_{0} \alpha_{v} \sin \theta-\frac{B}{A^{2}}\left(\alpha_{u}^{2}-\alpha_{v}^{2}\right) \sin \theta \cos \theta\right\} \\
0 & \frac{\alpha_{u} \alpha_{v}\left(1+\tan ^{2} \theta\right)}{C}
\end{array}\right) \# \\
& \boldsymbol{\mu}=\left(\begin{array}{cc}
\frac{1}{A}\left(v_{0} \sin \theta+\alpha_{v} \rho-\frac{B}{A^{2}} \alpha_{u} \cos ^{2} \theta\right) & \frac{\alpha_{v} \cos \theta}{A} \\
\frac{\alpha_{v} \tan \theta}{C} & 0
\end{array}\right. \\
& \left.\begin{array}{cc}
\frac{1}{A}\left(u_{0} \cos \theta+\alpha_{u} \rho-\frac{B}{A^{2}} \alpha_{v} \sin ^{2} \theta\right) & \frac{\alpha_{u} \sin \theta}{A} \\
\frac{-\alpha_{u} \tan \theta}{C} & 0
\end{array}\right) \#(40) \\
& \left\{\begin{array}{c}
A=\sqrt{\left(\alpha_{u} \sin \theta\right)^{2}+\left(\alpha_{v} \cos \theta\right)^{2}} \\
B=\alpha_{u} v_{0} \sin \theta+u_{0} \alpha_{v} \cos \theta+\alpha_{u} \alpha_{v} \rho \#(41) \\
C=\left(\alpha_{u} \tan \theta\right)^{2}+\alpha_{v}^{2}
\end{array}\right.
\end{aligned}
$$


Combine equations (35) and (38), the expression is shown below.

$$
\begin{gathered}
\omega \\
\dot{\sigma}
\end{gathered}=\mathrm{N}_{\text {rot }}^{+}\left(\lambda\left(\mathrm{L}_{\mathrm{i}} \mathrm{L}_{\mathrm{i}}^{*}\right) \lambda_{\text {trans }} \mu \mathrm{E}_{\text {trans }}\left(\tau \tau^{*}\right)\right) \#(42)
$$

where $\mathbf{N}_{\text {rot }}=\left(\boldsymbol{\eta} \mathbf{J}_{\text {rot }} \boldsymbol{\mu}\right)$ is a $2 \times 7$ matrix.

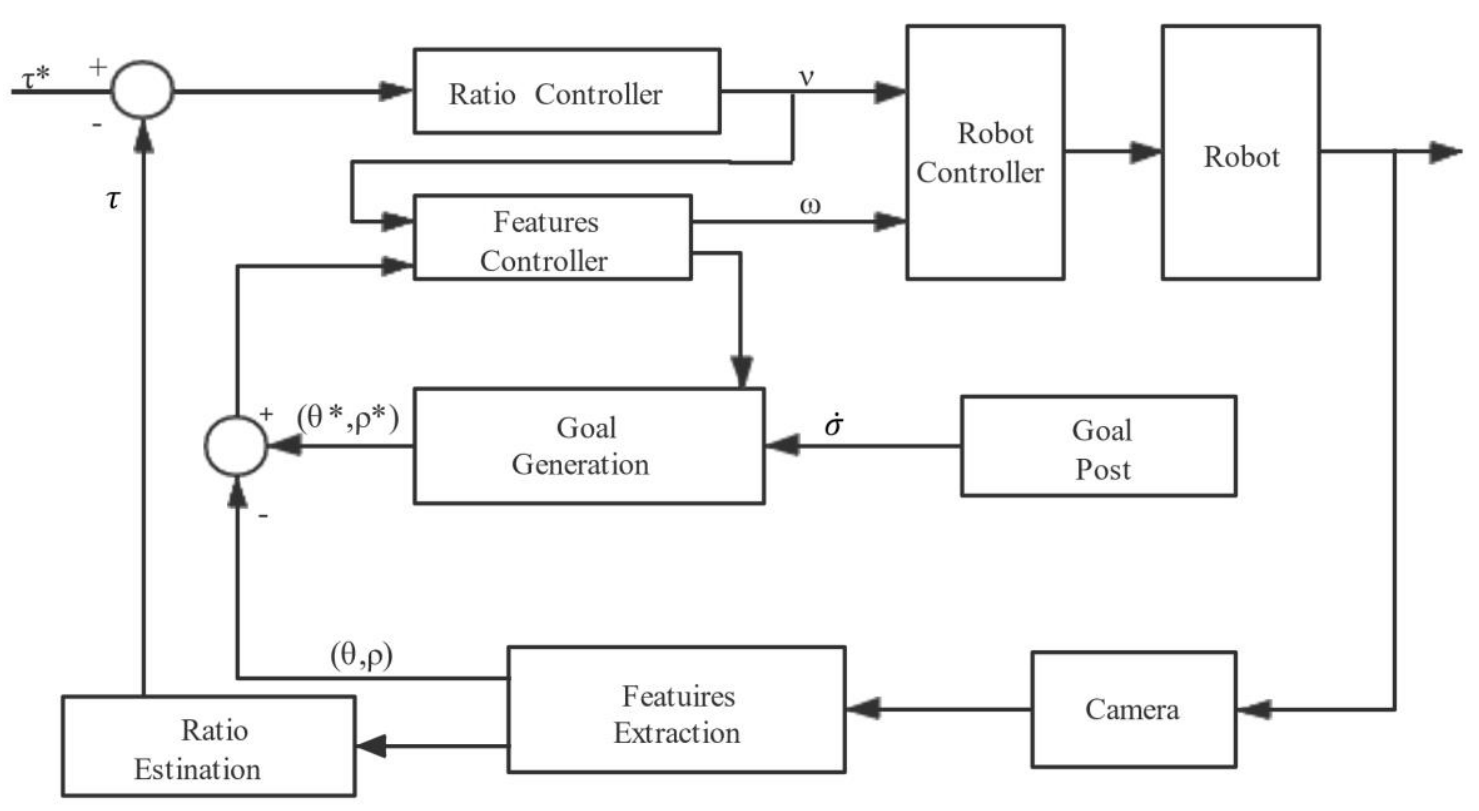

Fig 5 Block diagram of 3D reaching control system.

\section{PARAMETERS SETTING AND RESULTS}

In this section, a method of numerical simulation is used to evaluate the effectiveness of the proposed approach. The translational motion is restrained into the optimal trajectory. In order to control the optimal trajectory at every moment, the method does not constrain the rotation. Table 1 shows the distance ratio $\tau$ which is the present position and the target position from the origin of the camera frame to the reference plane of the object. The initial and target value of the straight-line parameters $\theta$ and $\rho$ in the image plane. In addition, the values of three parameters of a laser light calibration plane, which are a disparity angle $\alpha$, a tilt angle $\beta$ and an offset $Y_{0}$, are set to 85 degrees, 18 and $35 \mathrm{~cm}$, respectively. The rotation angle $\gamma$ of the rotation axis is set to 60 degrees, and the direction vector $\mathrm{u}$ of the axis of rotation is set to $(0.266,-0.321,0.908)$. The two gains $\lambda_{\text {rot }}$ and $\lambda_{\text {trans }}$ of rotational and 
translation controls are all set to 0.1 . The camera focal distance is set to $12 \mathrm{~mm}$.

Table 1 Motion parameters for visual reaching tasks

\begin{tabular}{|c|c|c|c|}
\hline values & angle $\boldsymbol{\theta}(\mathbf{d e g})$ & distance $\rho(\mathbf{c m})$ & ratio $\boldsymbol{\tau}$ (rate) \\
\hline initial values & 40.0 & 6.0 & 25.0 \\
\hline goal values & 20.0 & 12.0 & 1.0 \\
\hline
\end{tabular}

(A)Translational Velocity with Two Cases

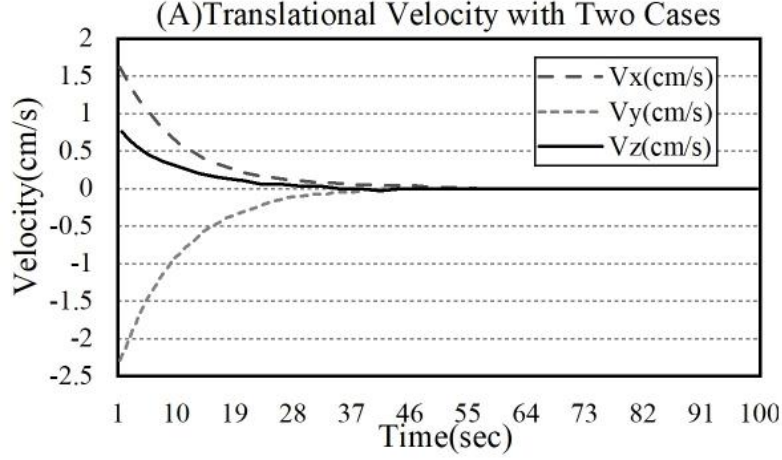

(C)Angle $\theta$ of Image Line with known Case

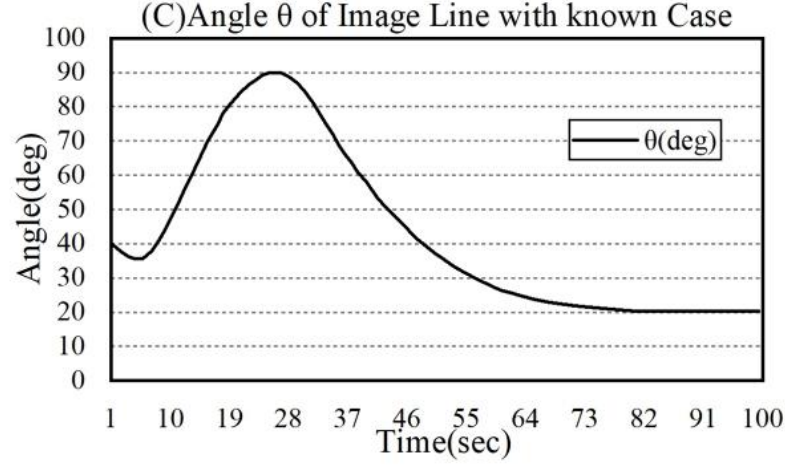

(E)Ratio $\tau$ of Distance with Two Cases

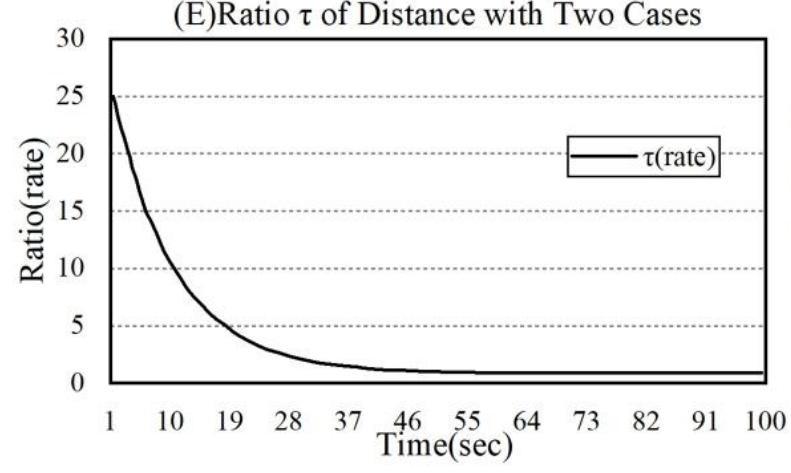

(B)Rotational Velocity with Known Case

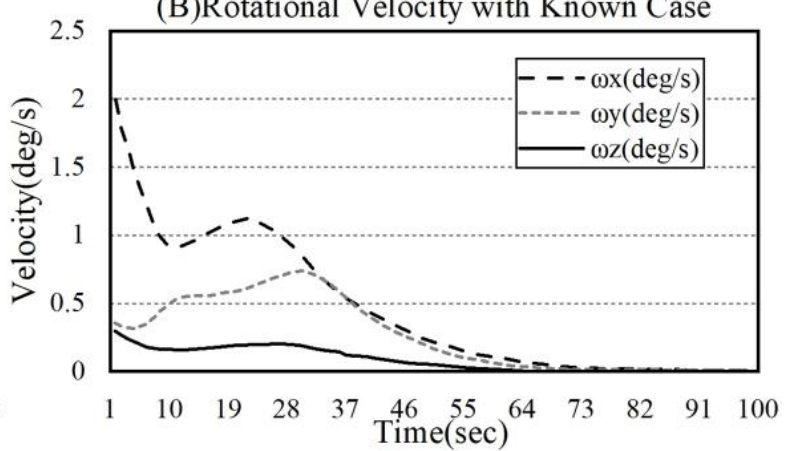

(D)Distance $\rho$ of Image Line with known Case

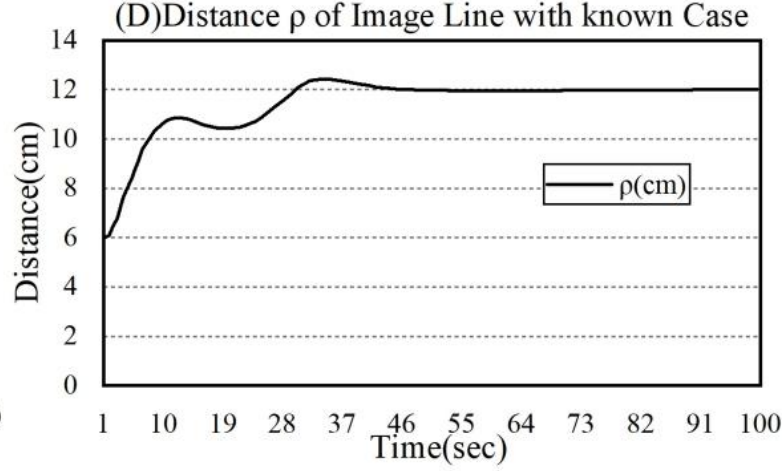

(F)Rotational Velocity with Unknown Case

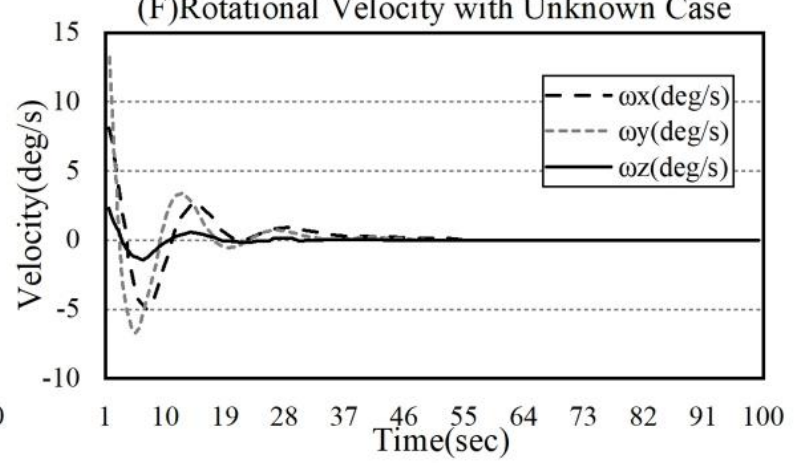


Fig 6 (A)-(F) Some results of visual servoing in 3D reaching tasks using quaternion.

The simulation was performed using two cases where the inside parameters of a camera are known and unknown. In the known case of the camera parameters, Fig.6 (A)-(E) shows the result of the simulation. Fig.6 (A) shows the changes in translational velocity. Fig.6 (B) shows the change of rotation velocity. What emerges from the above-mentioned figures reported here is that the velocity curves smoothly converge to zero. The translational velocity and rotational velocity converge in 55s and 75s, respectively. Fig 6 (C) and (D) show the changes of parameters $\theta$ and $\rho$ of the straight line in the image frame, which shows a stable convergence to 20 degrees and $12 \mathrm{~cm}$ in $82 \mathrm{~s}$ and $48 \mathrm{~s}$, respectively. Fig 6 (E) shows the distance ratio of the present and the goal positions from the camera's origin to the grasping plane of an object is converged to 1 in the 50th s, which means the goal position has arrived. Fig 6 (A) and Fig $6(\mathbf{F})$ show both the translational velocity and the rotation velocity are converged to zero in a short time due to the restraint of the translational movement is helpful to obtain the optimal straight-line trajectory.

For the case of unknown camera parameters, the straight-line parameters are dynamically estimated and presumed online simultaneously. First, the initial values of the camera parameters given from experience are shown in Table 2.

Table 2. Estimation of camera parameters

\begin{tabular}{|l|c|c|c|c|}
\hline camera & $\boldsymbol{\alpha}_{u}(\mathbf{m m})$ & $\boldsymbol{\alpha}_{v}(\mathbf{m m})$ & $\boldsymbol{u}_{\boldsymbol{0}}(\mathbf{c m})$ & $\boldsymbol{v}_{\boldsymbol{0}}(\mathbf{c m})$ \\
\hline initial parameters & & & & \\
\hline actual parameters & 15.0 & 14.0 & 7.0 & 6.0 \\
& 18.0 & 16.0 & 10.0 & 10.0 \\
\hline
\end{tabular}



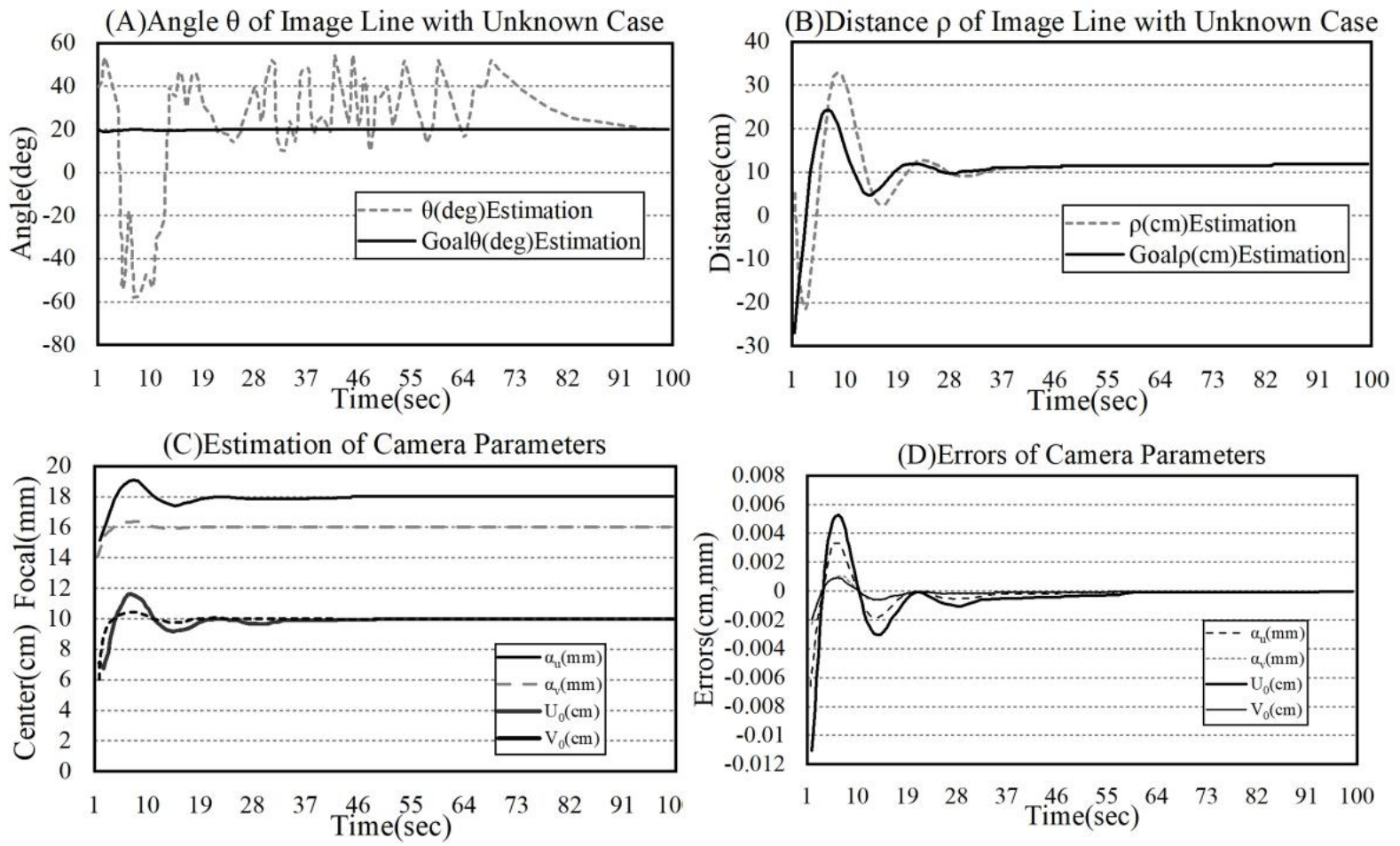

Fig 7 (A)-(D) Some results for the camera parameters are unknown.

According to Fig 7 (A), The parameter $\theta$ has a larger amplitude in the first 20 seconds. It exhibited an interval oscillation state between 20 seconds and 73 seconds and converged to the target value at the 95th second. Fig 7 (B) shows the parameter $\rho$ exhibits an alternating oscillation convergence state during the first 37 seconds and converges to the target value in the 40th second. Fig 7 (C) shows a focal length and the presumed values of the image center. In Fig 7 (C), a prompt convergence from the initial values to the target values is obsessed. Fig 7 (D) shows that the errors of image and the focal length are turned out zero. 

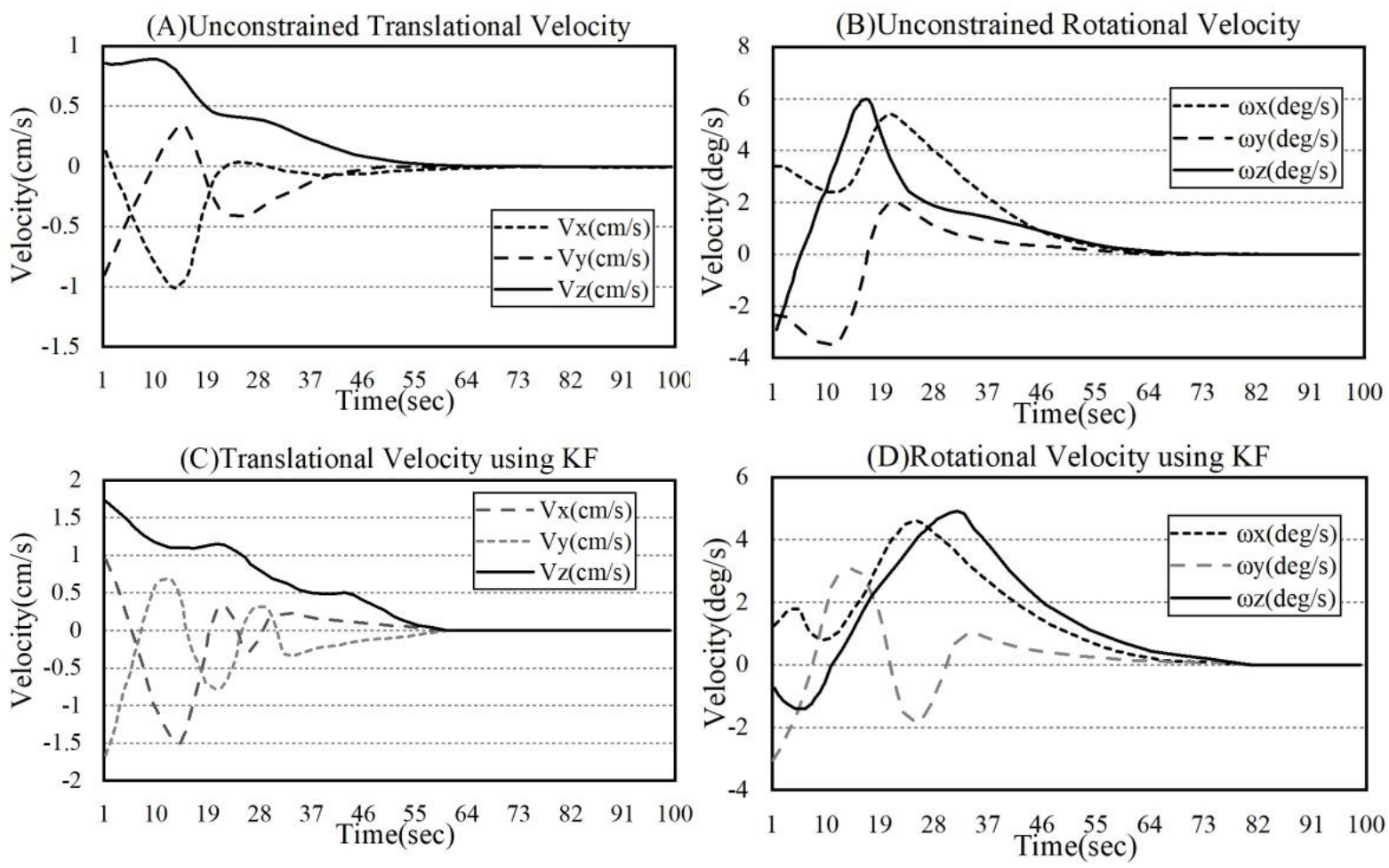

Fig 8 (A)-(D) Some results of 3D reaching tasks comparative algorithms.

Fig.8 (A) and (B) show the results of translational and rotation velocities using the conventional method where the translational motion is not restrained to an optimal trajectory. Fig.8 (A) shows the curvilinear trajectory which is not processed by our method in 3D space, and compared with this result, Fig.6 (A) shows the linear optimal trajectory. For comparison of the rotational velocity, Fig.6 (B) and Fig.8 (B) have similar results because the rotational motion is not restrained. Fig.8 (C) and (D) show the performance of the method of applying Kalman filter (KF) to the Jacobian matrix online identification in the translation speed and rotation speed, proposed by (Zhou et al. 2019). The results show that the translational velocity convergence time of the traditional IBVS and KF algorithms are 68 seconds and 64 seconds, respectively. The rate of change of the convergence curve of the KF algorithm is greater, while the traditional IBVS behaves relatively smoothly. Correspondingly, their rate of change in the convergence curve of the rotation speed is similar, and there is no significant difference in their convergence speed. Taken together, these results suggest that the proposed method is significantly higher than other comparative methods, and the method has attained 
convergence to a target position in the task space. In addition, the proposed method indicated the convergence range is also larger.

Table 3. The results of tracking error and routine run-time

\begin{tabular}{|c|c|c|}
\hline Method & $\begin{array}{c}\text { Average tracking } \\
\text { error (m) }\end{array}$ & $\begin{array}{c}\text { Average routine } \\
\text { run-time (s) }\end{array}$ \\
\hline Conventional IBVS & 0.0162 & 0.266 \\
\hline IBVS with KF & 0.0155 & 0.284 \\
\hline Ours & 0.0138 & 0.235 \\
\hline
\end{tabular}

Table 3 shows the results of tracking error and routine run-time. It can be concluded that under the same experimental conditions, compared with the traditional IBVS and Kalman filter algorithms, the average error of the algorithm proposed in this paper is smaller, which is outperform $14.1 \%$ and $11.0 \%$ respectively. The average execution time is shorter, better than $11.7 \%$ and $17.3 \%$ respectively. The quaternion IBVS algorithm not only takes into account the real-time performance of trajectory tracking but also has better tracking accuracy, which shows good application potential.

\section{CONCLUSIONS}

In this chapter, we proposed a novel visual servoing method for $3 \mathrm{D}$ reaching task. The features of the straight-line are extracted by the method of quaternion and then are applied to the restraint of the translational movement, aiming to acquire the optimal trajectory. In contrast with other conventional methods, the date of the 3D image Jacobian can be successfully estimated without the 3D model by the proposed method. Therefore, the approach can be used to avoid the respective drawbacks of classical position-based and image-based visual servoing. Our advantages of this approach are briefly summarized as follows: 
(1) In this system, since a reverse projection model is used, the 3D information of the object can be acquired without using a 3D model.

(2) The unit quaternion can map well with the 3D rotation. Therefore, the quaternion technique is particularly useful in rotational movement. It enabled the normal vector of a polyhedron can be properly settled.

(3) By this technique, the Jacobian data of 3D image as a surface parameter of a polyhedron is able to be presumed by using the quaternion and the distance information, which demonstrated the relationship between the surface parameter and the normal vector of the polyhedron in 3D space.

(4) In the scenario of $3 \mathrm{D}$ reaching task, to avoid the case that the object is disappeared from the view of camera, a method of restraint the translational movement is proposed to optimize the trajectory so that the camera can arrive at the target efficiently.

(5) In the situation that the interior parameters of a camera are unknown, the on-line identifying method still carried out the calibration of the camera simultaneously, according to the information of a straight-line in a way of presumption.

Simulation experiments with traditional IBVS and KF methods substantiate that the average convergence time of our method is 55 seconds, which is $19.1 \%$ and $14.1 \%$ superior to the above algorithms, respectively. In terms of running time, our method leads the other two algorithms by $11.7 \%$ and $17.3 \%$, and leads the other by $14.1 \%$ and $11.0 \%$ in tracking accuracy. In addition, our method can achieve stable tracking of the target point when the camera's internal parameters are unknown, which greatly expands the flexibility of the task. Overall, our method is superior to other comparison algorithms on convergence time, smoothness, and accuracy, demonstrating better accurate real-time tracking capabilities.

As a subject of future study, the tracking and stable grasping of moving objects will be considered. Furthermore, we will carry out the experiments in the next step for the verification of the present works. 


\section{ACKNOWLEDGEMENTS}

This research is supported by Special Fund for Bagui Scholars of the Guangxi Zhuang Autonomous Region, under Grand 2019A08; The Basic Ability Enhancement Program for Young and Middle-aged Teachers of Guangxi, under Grand 2020KY10018.

\section{REFERENCES}

Tsai D, Dansereau D G, Peynot T, et al. Image-based visual servoing with light field cameras[J]. IEEE Robotics and Automation Letters, 2017, 2(2): 912-919.

Mcfadyen A, Jabeur M, Corke P. Image-based visual servoing with unknown point feature correspondence[J]. IEEE Robotics and Automation Letters, 2016, 2(2): 601-607.

R. Ishiyama and K.Deguchi, "Optimal Motion Control for Image-Based Visual Servoing by Decoupling Translation and Rotation", J. of the Robotics Society of Japan, vol.17, no.5, pp.720-727, 1999.

E. Grosso, G.Metta, A.Oddera, and G.Sandini: "Robust visual servoing in 3D reaching tasks". IEEE Trans. Robot. Automat., vol.12, no.5, pp.732-741, Oct. 1996.

E. Malis, F.Chaumette, and S.Boudet: "2-1/2-D Visual servoing". IEEE Trans. Robot. Automat., vol.15, no.2, pp.238-250, April. 1999.

Sun X, Zhu X, Wang P, et al. A review of robot control with visual servoing[C]//2018 IEEE 8th Annual International Conference on CYBER Technology in Automation, Control, and Intelligent Systems (CYBER). IEEE, 2018: 116-121.

H. Fujimoto, L.C. Zhu, A. Sano, and S. Yamakawa, "Estimating image Jacobian of straight line based on multi-sensor," the 18th Annual Conf. of the Robotics Society of Japan, (in Japanese), vol.3, pp.1309-1310, Sept., 2000.

Fathian K, Jin J, Wee S G, et al. Camera relative pose estimation for visual servoing using quaternions[J]. Robotics and Autonomous Systems, 2018, 107: 45-62. 
Shen T, Yang J, Cai Y, et al. Visual servoing with cylinders: Reaching the desired location following a straight line[C]//2017 36th Chinese Control Conference (CCC). IEEE, 2017: $11183-11188$.

Liu J, Gao J, An X, et al. Autonomous Landing of an Unmanned Underwater Vehicle using Hybrid Visual Servoing Control with Image Moments and Quaternions[C]//OCEANS 2019-Marseille. IEEE, 2019: 1-6.

Hu G, Gans N, Dixon W. Quaternion-based visual servo control in the presence of camera calibration error[J]. International Journal of Robust and Nonlinear Control: IFACAffiliated Journal, 2010, 20(5): 489-503.

R. Horaud, F. Dornaika: "Hand-eye calibration". Int.J. Robot. Res. Vol.14. No.3, pp.195-210, June.1995.

S.J. Gordon, W.P. Seering: "Real-time part position sensing". IEEE Trans.Pattern Anal. Machine Intell., vol.10, no.3, pp374-386. May. 1988.

Wang N, He H. Adaptive homography-based visual servo for micro unmanned surface vehicles[J]. The International Journal of Advanced Manufacturing Technology, 2019, 105(12): 4875-4882.

Lee S, Jeong S, Hong Y D, et al. Image-based Visual Servoing Through Range and Feature Point Uncertainty Estimation of a Target for a Manipulator[J]. Journal of Institute of Control, Robotics and Systems, 2016, 22(6): 403-410.

Guo J, Zhu Z, Sun B, et al. A novel field box girder welding robot and realization of allposition welding process based on visual servoing[J]. Journal of Manufacturing Processes, 2020.

Keshmiri M, Xie W F. Image-based visual servoing using an optimized trajectory planning technique[J]. IEEE/ASME Transactions on Mechatronics, 2016, 22(1): 359-370.

Tian H, Cui Y, Minami M, et al. Frequency response experiments of eye-vergence visual servoing in lateral motion with $3 \mathrm{D}$ evolutionary pose tracking[J]. Artificial Life and 
Robotics, 2017, 22(1): 36-43.

Chen, D., Zhang, Y., Li, S., 2018. Tracking Control of Robot Manipulators with Unknown Models: A Jacobian-Matrix-Adaption Method. IEEE Trans. Ind. Inform. 14, 3044-3053.

Gu, J., Zhu, M., Cao, L., Li, A., Wang, W., Xu, Z., 2020. Improved Uncalibrated Visual Servo Strategy for Hyper-Redundant Manipulators in On-Orbit Automatic Assembly. Appl. Sci. 10, 6968.

Kosmopoulos, D.I., 2011. Robust Jacobian matrix estimation for image-based visual servoing. Robot. Comput.-Integr. Manuf. 27, 82-87.

Li, W., Xiong, R., 2021. A hybrid visual servo control method for simultaneously controlling a nonholonomic mobile and a manipulator. Front. Inf. Technol. Electron. Eng. 22, 141154.

Phanomchoeng, G., Rajamani, R., 2017. On the difference between bounded jacobian and lipschitz observers for nonlinear estimation applications. Trans. Can. Soc. Mech. Eng. $41,395-415$.

Song, X., Miaomiao, F., 2017. CLFs-based optimization control for a class of constrained visual servoing systems. ISA Trans. 67, 507-514.

Yang, L., Liu, Z., Wang, X., Xu, Y., 2019. An Optimized Image-Based Visual Servo Control for Fixed-Wing Unmanned Aerial Vehicle Target Tracking With Fixed Camera. IEEE Access 7, 68455-68468.

Zhou, Z., Zhang, R., Zhu, Z., 2019. Robust Kalman filtering with long short-term memory for image-based visual servo control. Multimed. Tools Appl. 78, 26341-26371.

Dirik M, Kocamaz A F, Dönmez E. Visual servoing based control methods for nonholonomic mobile robot[J]. Journal of Engineering Research, 2020, 8(2).

Kara T, Mary A H. Robust trajectory tracking control of robotic manipulators based on model-free PID-SMC approach[J]. Journal Of Engineering Research, 2018, 6(3). 